\title{
Mechanical Antagonism in Legged Robots
}

\author{
Andy Abate, Jonathan W. Hurst, and Ross L. Hatton \\ Robotics Program, School of Mechanical, Industrial, and Manufacturing Engineering \\ Oregon State University, Corvallis, Oregon 97331-6001 \\ Email: \{abatea, jhurst, ross.hatton\}@oregonstate.edu
}

\begin{abstract}
In this work, we use first principles of kinematics to provide a fundamental insight into mechanical power distribution within multi-actuator machines. Individual actuator powers-not their net sum-determine the efficiency and actuator size of a multi-joint machine. Net power delivered to the environment naturally discards important information about how that power is generated. For example, simultaneous positive and negative powers will cancel within a mechanism, wasting energy and raising peak power requirements. The same effect can bias power draw toward a single actuator while the other actuators do zero work. In general, it is best for all actuators to contribute equally to the net power demand because balance minimizes the mechanical power requirements of individual actuators. In this paper, we present the actuation power space, within which we measure the antagonism in a machine (joints working against each other due to kinematic constraints). We show the difference between the net power consumed by the task and the total power supplied by the actuators. We derive the power quality measure as a smooth objective function which encodes both antagonism and the balance of power between actuators. As a demonstration of our general framework, we apply our technique to a legged-robot design to find improved kinematics for performing a running gait. This technique finds mechanisms with optimal power distribution, regardless of actuator choice or loss models, so it can be applied early in the design process using mechanism kinematics alone. After choosing appropriate kinematics for an application, designers can independently optimize each actuator in a design to minimize local losses.
\end{abstract}

\section{INTRODUCTION}

It is often assumed that mechanical power output determines the power requirements of a machine. However, mechanical power requirements at the actuator level can be substantially greater than the net power requirement. Kinematic constraints may force some actuators to do work which opposes the task ${ }^{1}$ requiring other actuators do extra work to compensate. This degrades system performance and leads to larger requirements for the power supply and actuators.

In this paper, we show that antagonism ${ }^{2}$ can be avoided through the careful selection of mechanism kinematics relative to the task. As an example of this principle, consider a two-axis linear positioning table that performs a simple translation under load. Figure 1 a shows the power profile for a poor alignment with the task: one actuator produces excess power while the second actuator brakes (i.e. applies force opposite to

\footnotetext{
${ }^{1}$ The task is the trajectory of force-velocity pairs at the robot's end-effector (both linear and rotational) which requires some net mechanical power over time. The task can represent anything from lifting boxes to walking or running.

${ }^{2}$ We use the term antagonism as the extent to which one or more of a robot's joints do work contrary to the task: if the net power required by the task is positive, negative actuator powers are antagonistic, and vice versa.
}

a)
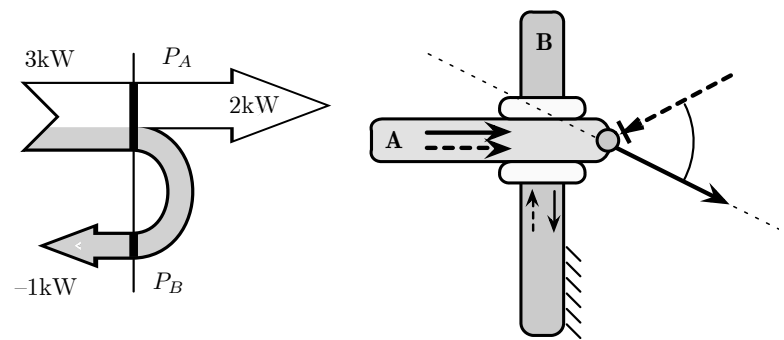

b)
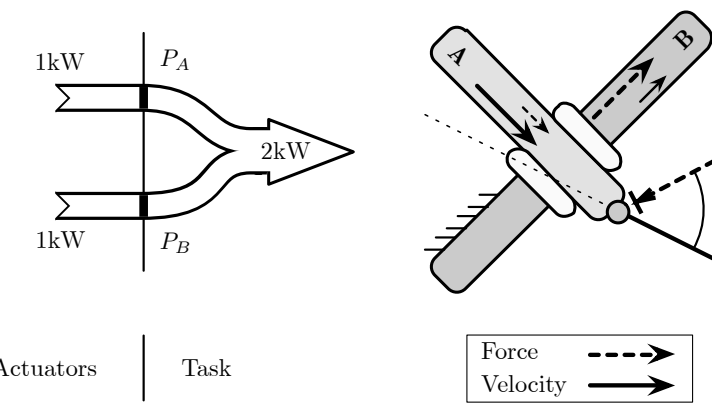

Figure 1. Configuration and mechanism choice can dramatically change the power requirements for an application. In this example, an $\mathrm{x}-\mathrm{y}$ table must resist the applied force while translating at a constant velocity. The device must hold its output on the path as well as push it along the path. While the task requires a positive net power $P_{\text {net }}=2 \mathrm{~kW}$, the kinematic constraints (i.e. the mechanism itself) in configuration (a) require that joint A generates $3 \mathrm{~kW}$ of power because joint $\mathrm{B}$ is dissipating $-1 \mathrm{~kW}$ of antagonistic power. Both the excess and removal of power will increase actuator power requirements and exacerbate conversion losses without contributing to the task. By adjusting the mechanism (in this case, rotating the table relative to the task), configuration (b) requires only $1 \mathrm{~kW}$ out of each joint by evenly balancing the $2 \mathrm{~kW}$ output.

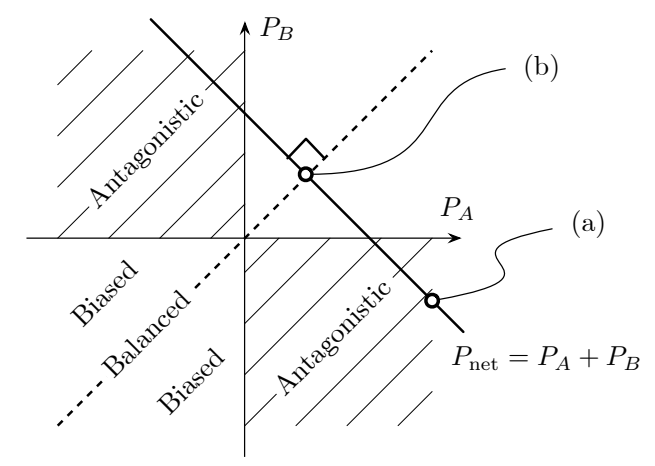

Figure 2. Plotting actuator powers in the power space shows how alignments (a) and (b) from Figure 1 move the operating point of the system and how that point is constrained to the net power line (or hyperplane in higher dimensions). Regions with opposing sign (hashed area) indicate antagonistic power use, and the primary diagonal indicates balanced power use $\left(P_{1}=P_{2}\right)$. This paper presents several measures on this space. 
its velocity). The alternative orientation in Figure $1 \mathrm{~b}$ balances the power output between both actuators, optimally delivering the required net power to the environment. This small configuration change lowers actuator A's power requirement by two-thirds. In more sophisticated applications, a similar change might only be possible during the design stage.

The operating points of the $\mathrm{x}-\mathrm{y}$ table can be plotted in what we call the power space (Figure 22). This space has one axis for each joint power and makes it easy to see when a point or trajectory contains antagonistic work. This is the $\mathrm{n}$-dimensional space in which we will measure the quantity of antagonism for any general n-DoF mechanism. In addition, we present the power quality measure as a principled, smooth, gradient-rich objective function for trajectories within this space, thereby ranking the mechanisms which generate them.

Performing a kinematic power quality optimization will minimize each joint's required mechanical power output, after which designers can optimally select motors and gearheads to minimize local losses 3 Power quality can be studied early in the design process before actuators are even specified.

Even when the cost of actuator antagonism is outweighed by other design features (e.g. workspace size, manipulability [20], maximum output force [11], or reduced complexity), any extra antagonistic power must still be accounted for. As such, understanding antagonism remains important for properly specifying a robot's actuation and power systems.

The following sections will discuss our definitions of the actuation power space, net power, and total power, quantify the amount of antagonistic work being performed in an n-DoF application, derive the power quality measure, and provide an example analysis for three different leg designs following a spring-mass $\stackrel{4}{4}^{4}$ running gait.

\section{RELATED WORK}

Antagonism can appear in any system with more than one actuator. It is present in high-DoF systems such as humanoid robots [8], and can even dominate the power requirements of some robots such as the compliant ATRIAS biped. For example, nearly all of the actuator power during the normal operation of ATRIAS is antagonistic due to the pantograph leg design [7]. During the stance phase of a walking or running gait, leg-length forces decompose into two equal and opposite joint torques, while the leg sweep requires equal joint motions. This combination of torque and speed leads to equal and opposite work from each actuator. Actual peak-to-peak mechanical actuator powers for an ATRIAS leg are 50\% larger than the task requires, as seen in Figure 3, and the unsigned work done by the actuators is $65 \%$ larger than necessary. In the ideal spring-mass case where the net power is zero, the pantograph leg design performs at zero efficiency and can waste an arbitrarily large amount of energy.

\footnotetext{
${ }^{3}$ For example, an electric motor behind a gearhead will require additional power to accelerate the rotor inertia, the motor has a resistive cost to generate torque, there are eddy losses in the iron, and the gearhead has friction.

${ }^{4}$ Reduced-order model which describes walking and running [3] 5].
}
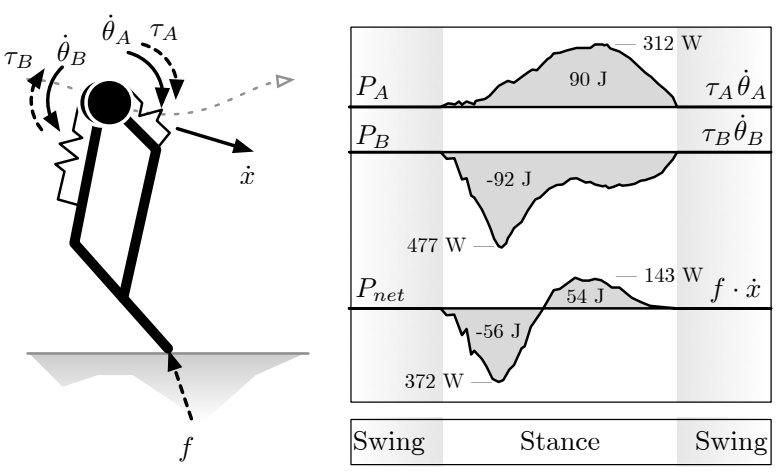

Figure 3. Real walking data from the ATRIAS biped $(1.5 \mathrm{~m} / \mathrm{s}, 60 \mathrm{~kg}$ robot $)$ shows that pantograph legs can amplify the power requirements for legged locomotion. Powers are calculated at the actuator output, so they exclude actuator losses and energy stored in the springs. For the sample step data, ATRIAS's actuators do -2 Joules of net work, joint A generates 90 Joules, and joint B dissipate 92 Joules. There is also a $50 \%$ increase in peak power requirements above the net power required by the gait.

Waldron and Kinzel [17] originally discussed the idea of "geometric work", which is based on the notion that backdriving actuators waste power. Song and Lee [14] noted that perhaps the purpose of a task is to remove energy, so actuator braking is sometimes favorable. With a negative net power, the forward-driven actuators are actually the undesirable component, rather than back-driven ones.

Internal power opposition is similar in concept to internal forces in parallel manipulators [19]. For example, an array of arms where each has a point of contact with a rigid object will have an inherent freedom in how to generate a net force on that object. In cases like this, the manipulators can apply arbitrary internal forces which cancel in their summation.

Titus and Spenny [16] use detailed electric and hydraulic motor models to find a machine's expected real efficiency. Other power optimizations find optimal motor-gearhead pairs for a joint's torque and speed trajectories [13, 12]. Motor power models will catch the effect of antagonism if applied to a multi-actuator system, because mechanical power is present in every motor model. Actuator selection and the implied loss models have no impact on their required mechanical power outputs, so optimization of individual actuators cannot improve a mechanism with low power quality.

Abate et al [1] used a special-case solution to antagonism for a spring-mass leg design where only one actuator performs work. The resulting mechanism has one leg-length actuator and one leg-angle actuator. During locomotion, the leg-length actuator resists the leg-length forces and the leg-angle actuator orients the leg, so only the leg-length actuator does work.

Manipulability scores [20, 10, 9] measure the ability of different kinematics to coordinate a set of joints in exerting force or moving through the environment. Interestingly, mechanisms with good manipulability scores can have arbitrarily poor power quality. For example, the pantograph mechanism in the ATRIAS leg has a manipulability ellipsoid with high isotropy, but requires the actuators to do equal and opposite work during stance (100\% antagonistic work). 


\section{Deriving Measures on the Power Space}

The antagonistic effects we are considering arise because the mapping from task to individual actuator powers inherently depends on the mechanism-induced actuator basis, even though the net power flux through the mechanism is independent of the choice of basis. This situation-which is analogous to how the $L_{1}$ norm depends on a choice of direction even though the $L_{2}$ norm is rotation invariant-has some subtle aspects, so in this section we provide a first-principles discussion of power calculations in mechanisms' actuator spaces.

\section{A. Actuation Tuples}

Kinematic constraints in a mechanism map the task's forces and velocities into actuator torques and speeds, thereby determining the individual power requirements of each actuator. For simplicity, we refer to forces and velocities in the work space as $f$ and $\dot{x}$, and those in the actuator space as $\tau$ and $\dot{\theta}$. This notation matches the respective translational and rotational nature of the spaces in our example systems, but this choice is not limiting; both sets of coordinates are fully-generalized forces and velocities.

The Jacobian matrix describes the mechanism kinematics at a point $\theta$ in its configuration space, and maps forces and velocities between the configuration and task spaces,

$$
\begin{aligned}
& J(\theta)=\left[\begin{array}{llll}
\frac{\partial x}{\partial \theta_{1}} & \frac{\partial x}{\partial \theta_{2}} & \ldots & \frac{\partial x}{\partial \theta_{n}}
\end{array}\right] \\
& \tau=f J \\
& J \dot{\theta}=\dot{x},
\end{aligned}
$$

where $f$ and $\tau$ are row vectors (covectors) of force and torque components. We make no rank assumptions about the Jacobian; we only assume there exists a method for determining actuator speeds from the task velocity 5 Actuator torques are always defined for any rank.

Given the force $f(t)$ and velocity $\dot{x}(t)$ trajectories for a task, designers can find the torque $\tau(t)$ and speed $\dot{\theta}(t)$ trajectories in the configuration space. When using the physical actuator basis, the components of these vectors represent the torque and speed of individual actuators,

$$
\begin{aligned}
& \tau(t)=\left(\tau_{1}(t), \tau_{2}(t), \ldots, \tau_{n}(t)\right) \\
& \dot{\theta}(t)=\left(\dot{\theta}_{1}(t), \dot{\theta}_{2}(t), \ldots, \dot{\theta}_{n}(t)\right) .
\end{aligned}
$$

Round braces are used to distinguish vectors from tuples, where the elements of a tuple refer to physical actuator quantities, and elements of a vector are an artifact of the basis used to measure that vector.

The element-wise product ${ }^{6}$ of torque and speed using the physical actuator basis gives the actuation power tuple,

$$
\begin{aligned}
\mathbf{P}(t)=\tau(t) \circ \dot{\theta}(t) & =\left(\tau_{1} \dot{\theta}_{1}, \tau_{2} \dot{\theta}_{2}, \ldots, \tau_{n} \dot{\theta}_{n}\right) \\
& =\left(P_{1}(t), P_{2}(t), \ldots, P_{n}(t)\right),
\end{aligned}
$$

\footnotetext{
${ }^{5}$ For redundant mechanisms, there can be many sets of joint speeds which result in the same end-effector velocity. This does not limit our analysis, but it does require an additional motion-planning step to select a unique joint trajectory for evaluation (see Section [IV] and Section VII).

${ }^{6}$ a.k.a. the Hadamard product: $a \circ b=\left(a_{1} b_{1}, a_{2} b_{2}, \ldots, a_{n} b_{n}\right)$
}

i.e., the set of powers flowing into or out of each actuator.

We call the space of all possible power tuples the power space. Tuples are plotted as points within this space.

\section{B. Net Power, Total Power, and Antagonism}

Net and total power measures on the power space can be combined to measure the quantity of antagonism in a power tuple. We use the net power as a measure of task energy requirements and the total power as a measure of how mechanical energy is being used within the mechanism.

Net power is the signed power flowing to the task. This power is equal to the sum of the terms in the power tuple, and also to the dot product of the force and velocity in either the work space or the configuration space,

$$
P_{\text {net }}(\mathbf{P})=\Sigma P_{i}=f \cdot \dot{x}=\tau \cdot \dot{\theta} .
$$

We define total power as the measure of energy flowing through the actuators. It is calculated as the unsigned sum (the $L_{1}$ norm) of the tuple powers,

$$
P_{\text {total }}(\mathbf{P})=\Sigma\left|P_{i}\right| .
$$

Unlike the force-velocity product in the net power (which is coordinate-invariant), the total power calculation inherits a coordinate dependence from the $L_{1}$ norm. Consequently, it takes on different values depending on the chosen kinematics. $P_{\text {total }}$ is never less than the magnitude of $P_{\text {net }}$, but can be considerably greater for some applications.

Any power which does not flow to the task appears twice in the total power: once to be emitted and once to be absorbed. Accordingly, the antagonistic power can be written as half the difference between total and normed net power,

$$
P_{\text {ant }}(\mathbf{P})=\frac{1}{2}\left(\Sigma\left|P_{i}\right|-\left|\Sigma P_{i}\right|\right)=\frac{1}{2}\left(P_{\text {total }}-\left|P_{\text {net }}\right|\right) .
$$

If all actuators are performing same-sign work, total and net power will be the same and cancel. Or, if any actuators are performing opposite-sign work, the total power will be greater than the net and the antagonistic power will be non-zero.

Figure 4 plots net power, total power, and antagonistic power over the actuation power space in $\mathbb{R}^{2}$. Note that antagonism only exists in regions where some elements in the power tuple have opposing signs. In $\mathbb{R}^{2}$, these are quadrants II and IV. Antagonism is zero in the regions containing the 1-tuple's span (power tuple of ones, quadrants I and III). There are only two such regions in the power space, regardless of dimension. As robots gain more degrees of freedom, there are more possible permutations of opposite-sign powers.

\section{Power Quality}

The basic antagonism measure does not make a good objective function for two reasons: first, it has a discontinuous first derivative; and second, regions where antagonism is zero are entirely level. In the level regions, there is no gradient information for an optimizer to follow. While these regions are technically antagonism-free, there is a robustness benefit for being further away from the antagonistic regions (see the analysis results in Section V. 

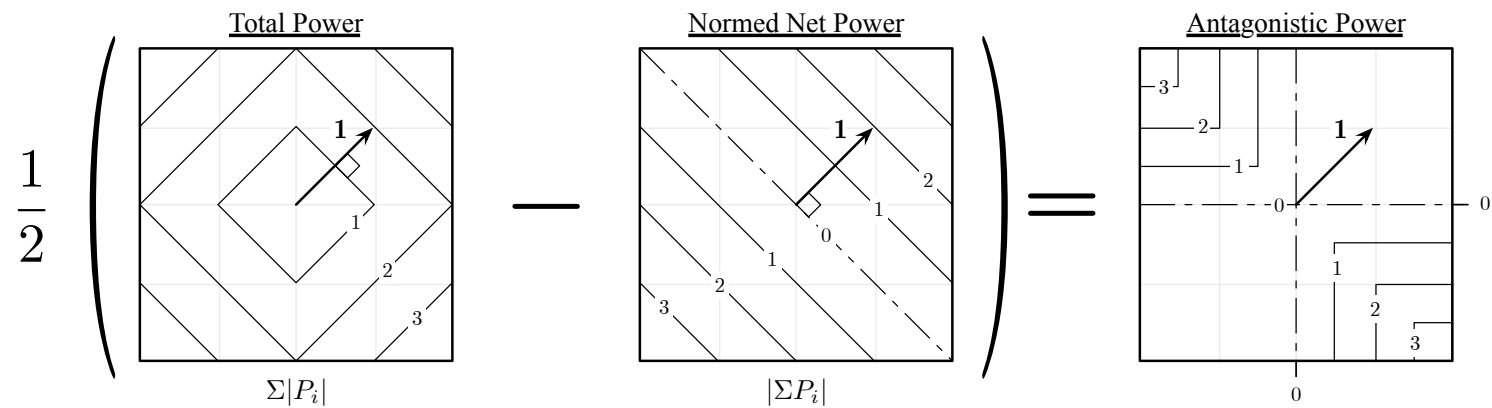

Figure 4. The total, net, and antagonistic power measures in the $\mathbb{R}^{2}$ power space. Net power is required by the task, total power represents the quantity of power use within the mechanism, and antagonism is a measure of the excess power being used inside the system. The 1-tuple is an important axis, because it defines the two regions (in any dimension) where antagonism is zero.
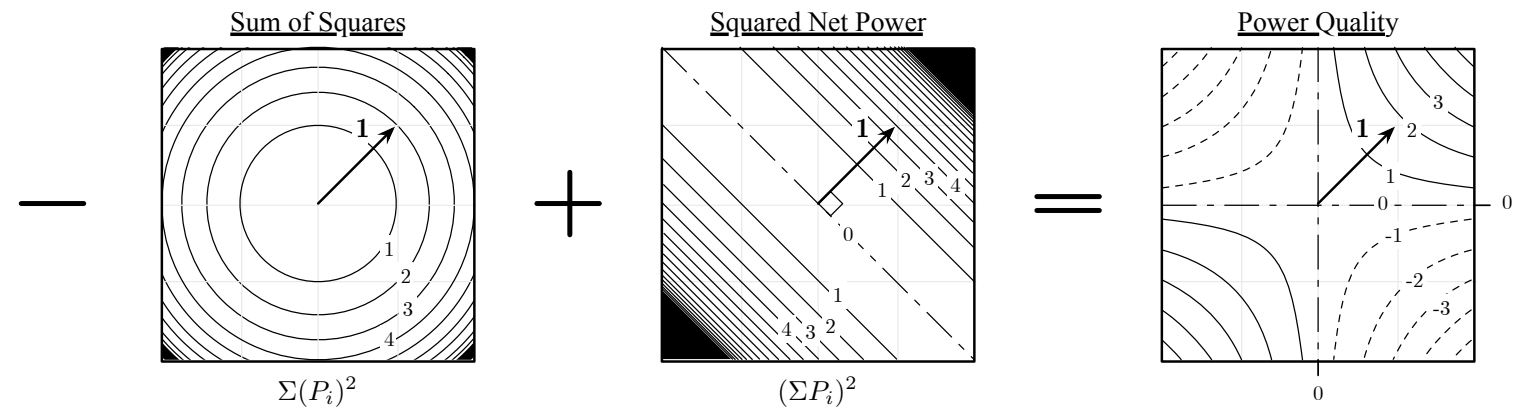

Figure 5. The power quality formula in two dimensions reduces to the product $2 P_{1} P_{2}$, which is a saddle centered at zero. Maximizing the power quality will minimize antagonism with solutions $P_{1}^{*}=P_{2}^{*}$. The power quality is an effective objective function due to its smoothness and gradient information, even in the regions of zero antagonism.

Following the derivation in Appendix A, we define the power quality measure as the difference between the squared sum of powers and the sum of squared powers,

$$
Q(\mathbf{P})=\left(\Sigma P_{i}\right)^{2}-\Sigma\left(P_{i}^{2}\right) .
$$

Note the similarity of the power quality to the antagonism measure by squaring terms instead of normalizing terms. The power quality is a polynomial function in $\mathbf{P}$, has rich gradient information, and has $C^{\infty}$ continuity. Maximizing this function minimizes antagonism and balances power. As is demonstrated in Appendix B, constrained maximization of the power quality results in the equal distribution of power among all actuators,

$$
\mathbf{P}^{*}=\left(\frac{P_{\text {net }}}{n} \frac{P_{\text {net }}}{n} \ldots \frac{P_{\text {net }}}{n}\right)=\frac{P_{\text {net }}}{n} \mathbf{1},
$$

where $\mathbf{1}=(1,1, \ldots, 1)$ is the 1 -tuple (the sum of the positive basis vectors of the power space).

In $\mathbb{R}^{2}$, the power quality is a saddle function centered at zero, where same-sign powers have positive values and opposite-sign powers have negative values,

$$
Q\left(P_{1}, P_{2}\right)=\left(P_{1}+P_{2}\right)^{2}-\left(P_{1}^{2}+P_{2}^{2}\right)=2 P_{1} P_{2} .
$$

Figure 5 shows the power quality measure in $\mathbb{R}^{2}$.

\section{OPTIMIZATION STRATEGY}

Using the power quality measure, our design process searches over the space of mechanism topologies for kinematics which are aligned with a given task. Each point in the search space will be a mechanism-task pair (or 'application') $(J(\theta), f(t), \dot{x}(t))$ and produce a trajectory $\mathbf{P}(t)$ in the actuation power space. If there are redundant mechanisms in the search space, there must also be a control law for determining a unique joint trajectory. Alternatively, the optimizer could search for the joint trajectory which produces the best power trajectory for that mechanism.

Selecting a mechanism/inverse kinematics law for a task uniquely determines the torques and speeds at each joint. Designers can then optimize each actuator's motor-gearhead pair in isolation, using the torque-speed trajectory as the task and treating the actuator as a $1-\mathrm{DoF}$ system. This method reduces the search space for a full design from $K+N M$ dimensions to one search across $K$ dimensions and $N$ searches across $M$ dimensions, where $K$ is the dimensionality of potential mechanisms, $N$ is the number of actuators, and $M$ is the dimensionality of the actuator designs. For example, searching over 20 mechanisms with two motor-gearhead pairs chosen out of 100 motors and 100 gearheads would traditionally require $(20 \times 100 \times 100 \times 100 \times 100)=2 \times 10^{9}$ evaluations. Our method uses only $(20)+(100 \times 100)+$ $(100 \times 100)=20 \times 10^{3}$ evaluations. 


\section{Design Evaluation}

As an example study, we consider the ability of legged robots to efficiently perform a running gait along a spring-mass trajectory [5]. We show a correlation between power quality and a robot's robust efficiency and peak actuator power, and we show how power quality can distinguish two designs which seem equally efficient. Although we simulate the efficiency of these designs, only the power quality is required in practice, because it is an indicator for these other features.

We evaluate three hand-picked mechanisms (Figure 6a). First, a parallel design similar to the ATRIAS leg design, with each link being driven relative to the hip. Second, a serial design with two links, a hip actuator, and a knee actuator. Third, a serial-link mechanism with a hanging center of mass which we call the spider design; the hip joint is elevated by $40 \mathrm{~cm}$ relative to the other designs, and link lengths are increased by $20 \mathrm{~cm}$ to compensate. Forces at the toe continue to be directed through the CoM, but the line of action no longer intersects the hip.

For the purposes of our example, we investigate rigid, massless mechanisms only ${ }^{7}$ There is no energy stored within the mechanisms. We also ignore the kinetic energy lost to inelastic collisions with the ground and any frictional losses.

We select a human-like gait with a peak vertical force of just above $2 \mathrm{x}$ body weight and a peak-to-peak vertical CoM displacement of roughly $7 \mathrm{~cm}$. Table I provides parameters for generating the spring-mass gait which the robots follow. This gait has the robots running at an average speed of $2.9 \mathrm{~m} / \mathrm{s}$ (9-minute mile), taking roughly 1-meter strides. We have selected an equilibrium gait, so each step will be identical to the last and we only need to simulate a single one.

For each design, we use inverse kinematics to determine its actuator torques and speeds. There are many methods for finding joint positions for redundant mechanisms [6, 18. 15], but in this case, we use constrained minimization via MATLAB's fmincon function. We find the actuator power trajectories for each design as follows:

$$
\begin{aligned}
\text { Design kinematics: } & x=X(\theta), J=\partial X / \partial \theta \\
\text { Task trajectory: } & x(t), f(t) \\
\text { Solve for joint positions: } & \theta(t) \text { s.t. } X(\theta(t))=x(t) \\
\text { Joint torques: } & \tau_{i}(t)=f_{i}(t) J(\theta(t)) \\
\text { Joint velocities: } & \dot{\theta}_{i}(t)=\mathrm{d} \theta_{i}(t) / \mathrm{dt} \\
\text { Joint powers: } & P_{i}(t)=\tau_{i}(t) \dot{\theta}_{i}(t)
\end{aligned}
$$

We estimate the true energy consumption of each design with a simple, zero-regeneration motor model that discards negative power,

$$
\begin{array}{r}
P_{\text {req }}(t)=\sum \max \left(P_{i}(t), 0\right) \\
E=\int_{0}^{T} P_{\text {req }}(t) \mathrm{dt} .
\end{array}
$$

\footnotetext{
${ }^{7}$ When applying this analysis to a compliant mechanism, actuator powers and antagonism continue to be calculated using torques and speeds at the actuators' outputs, regardless of the dynamics of the mechanism.
}

Table I

GENERATIVE SPRING-MASS PARAMETERS

\begin{tabular}{lcc}
\multicolumn{1}{c}{ Quantity } & Value & Units \\
\hline \hline Mass & 75 & $\mathrm{~kg}$ \\
Stiffness & $1.1 \times 10^{4}$ & $\mathrm{~N} / \mathrm{m}$ \\
Apex height & 0.93 & $\mathrm{~m}$ \\
Apex velocity & 3 & $\mathrm{~m} / \mathrm{s}$ \\
Neutral length & 1.0 & $\mathrm{~m}$ \\
Touchdown angle & 0.4 & $\mathrm{rad}$ \\
Gravitational constant & 9.81 & $\mathrm{~m} / \mathrm{s}^{2}$ \\
\hline \multicolumn{1}{c}{ RESULTING GAIT } & \\
\hline Peak Force & 1560 & $\mathrm{~N}$ \\
Stride Period & 0.3578 & $\mathrm{~s}$ \\
Stride Length & 1.036 & $\mathrm{~m}$ \\
Average Speed & 2.9 & $\mathrm{~m} / \mathrm{s}$ \\
CoM Peak-to-Peak & 0.0717 & $\mathrm{~m}$
\end{tabular}

Differences in kinematics lead to diverse power requirements (Figure 6p). The parallel design has the actuators fighting through the entirety of stance phase (i.e. they supply opposite-sign power). Because the parallel actuators fight each other, the actuator power requirements go up, peaking at $2.3 \mathrm{~kW}$ ( $1 \mathrm{~kW}$ above the peak power required by the task). The spider design has both actuators supplying the same sign of power always, because the line of action of the task force crosses the hip joint exactly when the motion of the knee joint reverses. Because the spider actuators assist each other, the actuator power requirements go down, peaking at $1.0 \mathrm{~kW}(0.3 \mathrm{~kW}$ below the peak net power $)$.

Using the mechanical power trajectories along with our minimal motor model, we calculate each robot's mechanical cost of transpor ${ }^{8}$ (MCoT). We find that the parallel design requires 330 Joules per step (220 Joules in excess of the required task work) and both the serial and spider designs require 110 Joules per step (which is bounded by the net task work of 110 Joules). These correspond to MCoTs of 0.43 and 0.14 , respectively 9

Even when the serial and spider designs are equal in MCoT, the spider sees a robustness benefit when we consider variations in the power-space trajectories (Figure 6c). If the nominal trajectory rides the line between antagonistic and cooperative power regions (as with the serial design) these power variations can bring the system into an antagonistic region, causing the average MCoT to increase. If the nominal trajectory is in a cooperative region (as with the spider design) it is likely to stay there and experience less variation in the step cost. For random offsets of $\pm 1 \mathrm{rad} / \mathrm{s}$ to the actuators' speeds and $\pm 100 \mathrm{~N}$-m to the actuators' torques, we find that the average MCoT is least for the spider design, and that it has the smallest standard deviation in MCoT.

\footnotetext{
${ }^{8}$ The energy required to move a weight a unit distance $(E / m g d)$.

${ }^{9}$ For reference, a human has a mechanical cost of transport of 0.055 [4]. Running at roughly $2.9 \mathrm{~m} / \mathrm{s}$, a $75 \mathrm{~kg}$ human requires an average positive work of 42 Joules per step. The leg designs have a task with a higher energy bound, because humans benefit from storing elastic energy to help offset the cyclic work requirements in walking and running [2].
} 

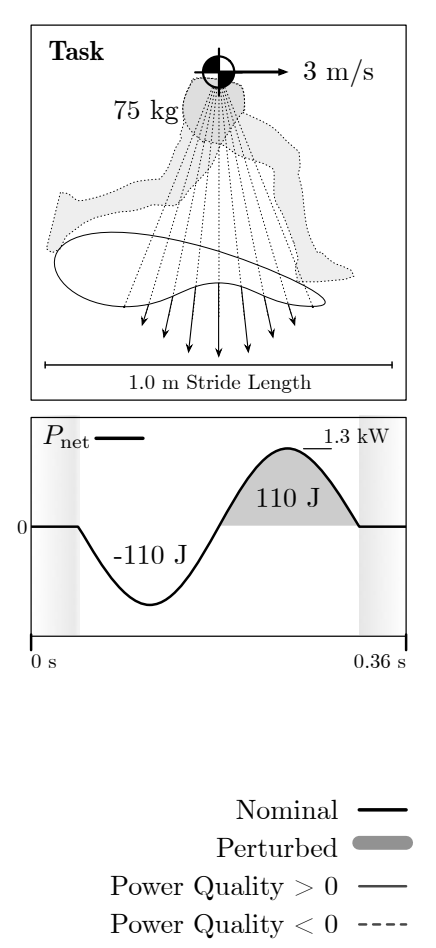

Power Quality $<0$

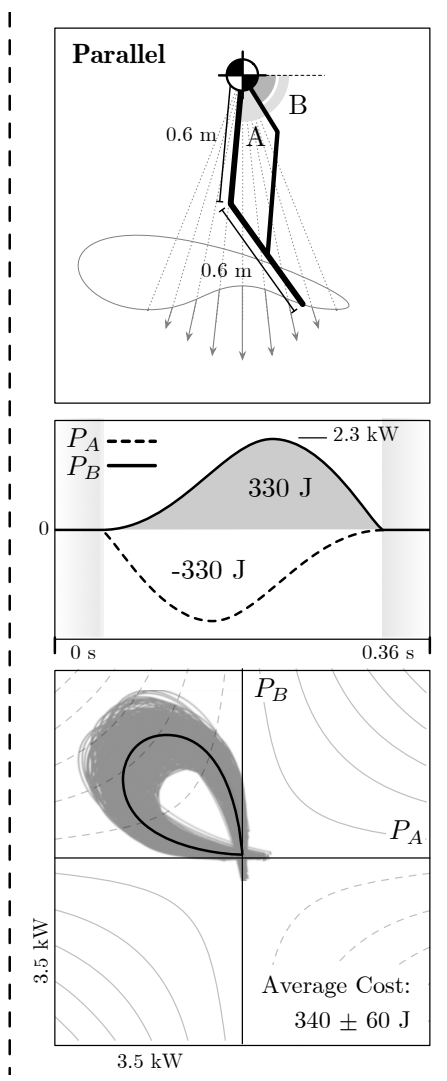

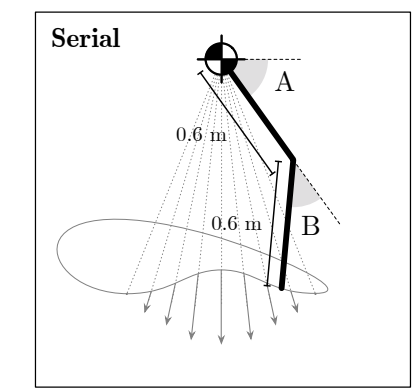
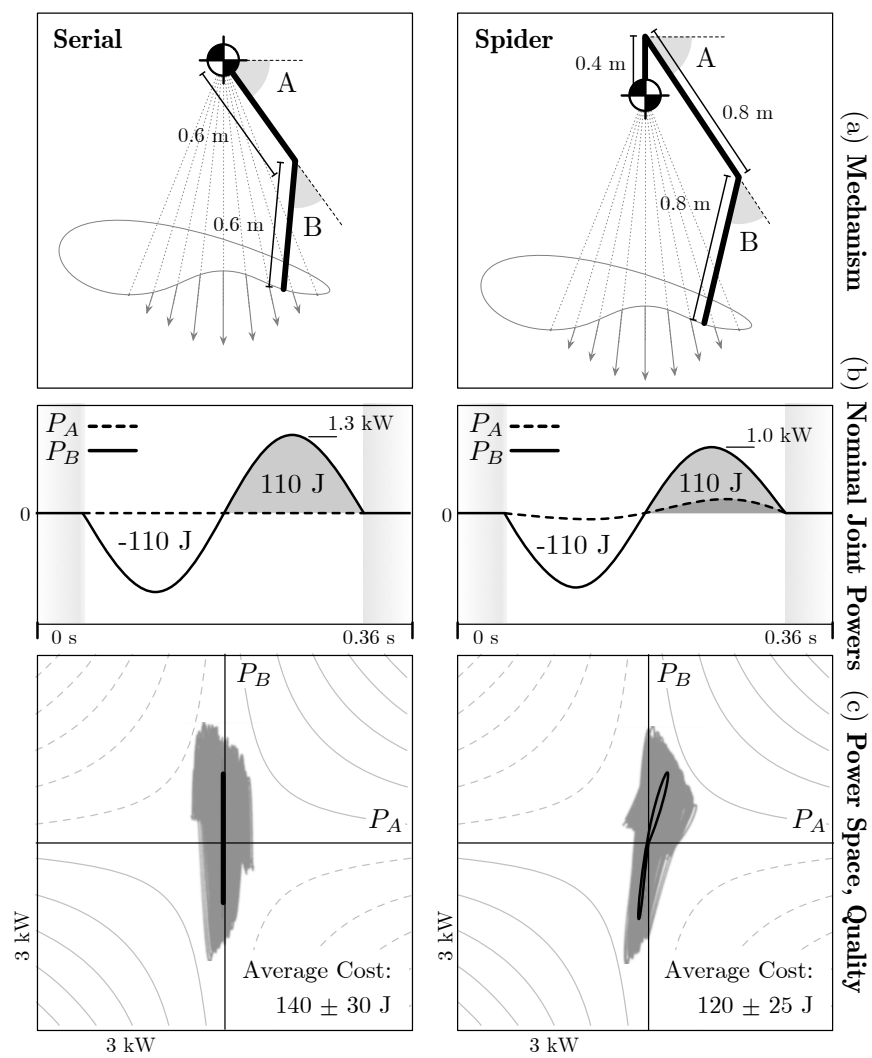

Figure 6. (a) Three candidate leg designs perform a single running gait with a variety of (b) resulting mechanical power requirements. The parallel design is the least balanced in terms of power use, the serial design is biased toward a single actuator, and the spider design's actuators share the net power generation. The simulation uses a basic motor model to estimate the step cost, which is represented by the positive area under each power trajectory. The step cost will fluctuate when (c) the task is perturbed, leading to an average step cost along with a standard deviation in cost. The farther a power trajectory travels through an area with high power quality, the better the power sharing, peak power requirements, and resulting step cost will be.

Table II

RESULTS

\begin{tabular}{lrrrc}
\multicolumn{1}{c}{ Quantity } & Parallel & Serial & Spider & Units \\
\hline \hline Nominal peak power & 2.3 & 1.3 & $\mathbf{1 . 0}$ & $\mathrm{kW}$ \\
\hline Nominal MCoT & 0.43 & $\mathbf{0 . 1 4}$ & $\mathbf{0 . 1 4}$ & $\cdot$ \\
Average MCoT & 0.45 & 0.18 & $\mathbf{0 . 1 6}$ & $\cdot$ \\
StdDev MCoT & \pm 0.08 & \pm 0.04 & $\pm \mathbf{0 . 0 3}$ &. \\
\hline Mean power quality & $-2,600$ & 0.00 & $\mathbf{1 5 0}$ &.
\end{tabular}

Table II shows the mutual correspondence of mean power quality with the nominal peak power, nominal MCoT, average MCoT, and MCoT variation for the three designs in this study. The actuator coordination seen in the spider design is not something that appears when looking at the MCoT, but it is captured by the power quality.

Even though we have found a mechanism with high-quality kinematics, the design is not complete without specifying motors and gearheads for the actuated joints. We end our analysis here, however, as there is already a large volume of knowledge dedicated to loss models and actuator optimization.

\section{Conclusions}

Peak power demand and efficiency are driven by the individual actuators, not the net power requirements of the task. We present the actuation power space and the power quality measure as tools for understanding and optimizing a design's kinematic power distribution, thereby minimizing the work required out of each actuator. Understanding the effect of the mechanism kinematics is critical, because no amount of actuator optimization can remove antagonism from a system or re-balance mechanical power.

Antagonism can be minimized by selecting mechanism kinematics which are appropriate for a given task. In our example, this is done using the power quality as an objective function for candidate designs. We show how antagonism affects the energetic cost of legged robots performing a spring-mass gait, both in their mechanical cost of transport and the power-sharing between actuators. By sharing power between the actuators, the spider design has lower actuator power requirements and is more energetically robust to variations in the gait.

Antagonism represents an important, fundamental feature of the kinematic constraints of mechanisms. The power quality metric is simple to calculate, but can yield large benefits when informing a mechanical design. 


\section{FUTURE WORK}

While we did not explicitly consider redundant manipulators in this work, it is possible that the added freedom of choosing joint velocities would allow a robot to avoid antagonism for a larger set of tasks, maybe even all tasks. For every additional degree of freedom beyond those needed to span the task space, there will be one additional degree of freedom in the null space of the mechanism kinematics. This could allow for the power tuple to be programmatically moved through the net power plane in real-time, such that it is always near the optimal distribution of powers among all the robot's actuators.

We also did not consider the effect of inertial forces on a rotating trunk, but it is likely that using a pitching torso could better balance the actuator powers. Such a design might have three links, like the spider design, but use a forward center of mass so that the hip and knee work together to extend and retract the virtual leg between the CoM and the toe. With equal torques and speeds for each actuator, this design would evenly distribute the task power. It is possible that this is why some animals incorporate a pitching cycle into their gait.

\section{ACKNOWLEDGEMENTS}

This material is based upon work supported by the National Science Foundation under Graduate Research Fellowship Program Grant No. 1314109 and under Grant No. 1462555.

\section{APPENDIX A: POWER QUALITY DERIVATION}

We derive our target from a function of the distance $r$ from the origin and angle $\phi$ from the 1-tuple in the actuation power space:

$$
q=r^{2} \cos (2 \phi),
$$

where $r^{2}$ is used instead of $r$ to ensure smoothness. Remembering that $\phi$ is the angle from the 1-axis, the cosine of $2 \phi$ has high points along the 1-axis and low points perpendicular to the 1 -axis. We can rewrite this equation in terms of components of the power tuple $P_{i}$. For a power space of dimension $n$, let us define the 1-tuple as

$$
\mathbf{1}=(1,1, \ldots, 1), \quad|\mathbf{1}|=\sqrt{n} .
$$

We can get any power tuple's angle from the 1-tuple via the dot-product and its distance from origin via its magnitude:

$$
\begin{aligned}
& \phi=\cos ^{-1}\left(\frac{\mathbf{1} \cdot \mathbf{P}}{|\mathbf{1}||\mathbf{P}|}\right)=\cos ^{-1}\left(\frac{\Sigma P_{i}}{\sqrt{n} r}\right) \\
& r=\sqrt{\Sigma\left(P_{i}^{2}\right)} .
\end{aligned}
$$

Plugging these terms into the power quality and reducing the cosine-anticosine term, we find

$$
\begin{aligned}
q(\mathbf{P}) & =r^{2} \cos \left(2 \cos ^{-1}(\cdot)\right)=r^{2}\left[2(\cdot)^{2}-1\right] \\
& =2 r^{2}\left(\frac{\Sigma P_{i}}{\sqrt{n} r}\right)^{2}-r^{2} \\
& =\frac{2}{n}\left(\Sigma P_{i}\right)^{2}-\Sigma\left(P_{i}^{2}\right) .
\end{aligned}
$$

The $2 / n$ arrives because the coordinate axes for dimensions higher than two are not $45^{\circ}$ from the 1-axis, making $\cos 2 \phi$ nonzero along the axes. Antagonism engages at the coordinate axes in power space, and we desire the same behavior from the power quality, hence we remove the $2 / n$. This is simple enough to prove by looking at the case where only one power in the tuple is nonzero: $\left(\Sigma P_{i}\right)^{2}-\Sigma\left(P_{i}{ }^{2}\right)=P_{j}{ }^{2}-P_{j}{ }^{2}=0$. This would not be the case in general if we had included the $2 / n$ term.

\section{APPENDIX B: MINIMIZATION EQUIVALENCE}

Maximizing a machine's power quality will inherently minimize the sum of squares of actuator powers and make the actuator requirements as low as physically possible. We can show this by solving two minimization problems, one for least-squares and one for the power quality. First, we solve for the least-squares power subject to the net power constraint:

$$
\begin{aligned}
\min . & f(\mathbf{P})=P_{1}{ }^{2}+P_{2}{ }^{2}+\cdots+P_{n}{ }^{2} \\
\text { s.t. } & g(\mathbf{P})=P_{1}+P_{2}+\cdots+P_{n}-P_{\text {net }}=0 .
\end{aligned}
$$

As discussed, the constraint function represents a hyperplane perpendicular to the 1-axis. The sum of squared joint powers will have spherical level-sets in power space, so the solution to this minimization is a point coincident with both the hyperplane and the smallest possible hypersphere. These points are proportional to the 1-tuple. We can solve this minimization problem using the Lagrange Multiplier method:

$$
\begin{aligned}
\nabla f & =\lambda \nabla g \\
\nabla\left(\Sigma P_{i}{ }^{2}\right) & =\lambda \nabla\left(\Sigma P_{i}-P_{\text {net }}\right) \\
2 \mathbf{P}^{*} & =\lambda \mathbf{1} \\
\mathbf{P}^{*} & =\lambda \mathbf{1} / 2
\end{aligned}
$$

Solving for lambda by plugging $\mathbf{P}^{*}$ back into the constraint equation gives

$$
\begin{aligned}
g\left(\mathbf{P}^{*}\right)= & P_{1}+P_{2}+\cdots+P_{n}=P_{\text {net }} \\
= & n \lambda / 2=P_{\text {net }} \Longrightarrow \lambda=2 P_{\text {net }} / n \\
& \Longrightarrow \mathbf{P}^{*}=\frac{P_{\text {net }}}{n} \mathbf{1}
\end{aligned}
$$

Maximizing the power quality subject to the same constraint is the equivalent problem:

$$
\begin{aligned}
\min . & Q(\mathbf{P})=\Sigma\left(P_{i}{ }^{2}\right)-\left(\Sigma P_{i}\right)^{2}=f(\mathbf{P})-\left(\Sigma P_{i}\right)^{2} \\
\text { s.t. } & g(\mathbf{P})=\Sigma P_{i}-P_{\text {net }}=0 .
\end{aligned}
$$

which results in

$$
\begin{aligned}
\nabla Q & =\lambda \nabla g \\
\nabla\left(\Sigma P_{i}^{2}\right)-\nabla\left(\Sigma P_{i}\right)^{2} & =\lambda \nabla\left(\Sigma P_{i}-P_{\text {net }}\right) \\
2 \mathbf{P}^{*}-2\left(\Sigma P_{i}\right) \nabla\left(\Sigma P_{i}\right) & =\lambda \mathbf{1} \\
2 \mathbf{P}^{*}-2 P_{\text {net }} \mathbf{1} & =\lambda \mathbf{1} \\
\mathbf{P}^{*} & =\left(\lambda / 2+P_{\text {net }}\right) \mathbf{1}
\end{aligned}
$$


and again solving for lambda through the constraint equation

$$
\begin{aligned}
g\left(\mathbf{P}^{*}\right)= & \Sigma P_{i}=P_{\text {net }} \\
= & n\left(\lambda / 2+P_{\text {net }}\right)=P_{\text {net }} \Longrightarrow \lambda=2(1 / n-1) P_{\text {net }} \\
& \Longrightarrow \mathbf{P}^{*}=\frac{P_{\text {net }}}{n} \mathbf{1}
\end{aligned}
$$

which is the same solution we had for explicitly minimizing the sum of squared powers.

As a result, past optimizations which focus on the least-squares optimization of actuator powers will inherently minimize antagonism, whether or not that is the goal.

\section{REFERENCES}

[1] Andy Abate, Ross L Hatton, and Jonathan Hurst. Passive-dynamic leg design for agile robots. In Robotics and Automation (ICRA), 2015 IEEE International Conference on, pages 4519-4524. IEEE, 2015.

[2] R McNeill Alexander. Elastic energy stores in running vertebrates. American Zoologist, 24(1):85-94, 1984.

[3] Reinhard Blickhan. The spring-mass model for running and hopping. Journal of biomechanics, 22(11): 1217-1227, 1989.

[4] Steve Collins, Andy Ruina, Russ Tedrake, and Martijn Wisse. Efficient bipedal robots based on passive-dynamic walkers. Science, 307(5712):1082-1085, 2005.

[5] Hartmut Geyer, Andre Seyfarth, and Reinhard Blickhan. Compliant leg behaviour explains basic dynamics of walking and running. Proceedings of the Royal Society of London B: Biological Sciences, 273(1603):2861-2867, 2006.

[6] Andrew A Goldenberg, Beno Benhabib, and Robert G Fenton. A complete generalized solution to the inverse kinematics of robots. Robotics and Automation, IEEE Journal of, 1(1):14-20, 1985.

[7] Christian Hubicki, Jesse Grimes, Mikhail Jones, Daniel Renjewski, Alexander Spröwitz, Andy Abate, and Jonathan Hurst. Atrias: Enabling agile biped locomotion with a template-driven approach to robot design. International Journal of Robotics Research, 2016.

[8] A.D. Kuo. Choosing your steps carefully. Robotics Automation Magazine, IEEE, 14(2):18-29, June 2007. ISSN 1070-9932. doi: 10.1109/MRA.2007.380653.

[9] Imed Mansouri and Mohammed Ouali. The power manipulability-a new homogeneous performance index of robot manipulators. Robotics and Computer-Integrated Manufacturing, 27(2):434-449, 2011.

[10] Jean-Pierre Merlet. Jacobian, manipulability, condition number, and accuracy of parallel robots. Journal of Mechanical Design, 128(1):199-206, 2006.

[11] Ryuma Niiyama and Yasuo Kuniyoshi. Design principle based on maximum output force profile for a musculoskeletal robot. Industrial Robot: An International Journal, 37(3):250-255, 2010.

[12] Siavash Rezazadeh and Jonathan W Hurst. On the optimal selection of motors and transmissions for electromechanical and robotic systems. In Intelligent Robots and Systems (IROS 2014), 2014 IEEE/RSJ International Conference on, pages 4605-4611. IEEE, 2014.

[13] Fredrik Roos, Hans Johansson, and Jan Wikander. Optimal selection of motor and gearhead in mechatronic applications. Mechatronics, 16(1):63-72, 2006.

[14] S-M Song and Jong-Kil Lee. The mechanical efficiency and kinematics of pantograph type manipulators. In Robotics and Automation, 1988. Proceedings., 1988 IEEE International Conference on, pages 414-420. IEEE, 1988.

[15] Gaurav Tevatia and Stefan Schaal. Inverse kinematics for humanoid robots. In Robotics and Automation, 2000. Proceedings. ICRA'OO. IEEE International Conference on, volume 1, pages 294-299. IEEE, 2000.

[16] NA Titus and CH Spenny. Power metrics for robot planning and redundancy resolution. In Intelligent Control, 1994., Proceedings of the 1994 IEEE International Symposium on, pages 153-159. IEEE, 1994.

[17] KJ Waldron and GL Kinzel. The relationship between actuator geometry and mechanical efficiency in robots. Fourth, Symposium on Theory and Practice of Robots and Manipulators. Poland, 1981.

[18] Charles W Wampler et al. Manipulator inverse kinematic solutions based on vector formulations and damped least-squares methods. Systems, Man and Cybernetics, IEEE Transactions on, 16(1):93-101, 1986.

[19] David Williams and Oussama Khatib. The virtual linkage: A model for internal forces in multi-grasp manipulation. In Robotics and Automation, 1993. Proceedings., 1993 IEEE International Conference on, pages 1025-1030. IEEE, 1993.

[20] Tsuneo Yoshikawa. Manipulability of robotic mechanisms. The international journal of Robotics Research, 4(2):3-9, 1985. 